\title{
LncRNA CTD-2528L19.6 prevents the progression of IPF by alleviating fibroblast activation
}

Tingting Chen $\mathbb{1}^{1}$, Yingying Guo ${ }^{2,3}$, Jiayi Wang ${ }^{2,3}$, Liqiang Ai ${ }^{1}$, Lu Ma ${ }^{2,3}$, Wenxin He ${ }^{4}$, Zhixin Li ${ }^{4}$, Xiaojiang Yu ${ }^{2,3}$, Jinrui Li', Xingxing Fan ${ }^{5}$, Yunyan Gu $\mathbb{D}^{1}$ and Haihai Liang (D) $^{2,3,6}$

\begin{abstract}
Long non-coding RNAs (IncRNAs) have emerged as critical factors for regulating multiple biological processes during organ fibrosis. However, the mechanism of IncRNAs in idiopathic pulmonary fibrosis (IPF) remains incompletely understood. In the present study, two sets of IncRNAs were defined: IPF pathogenic IncRNAs and IPF progression IncRNAs. IPF pathogenic and progression IncRNAs-mRNAs co-expression networks were constructed to identify essential IncRNAs. Network analysis revealed a key IncRNA CTD-2528L19.6, which was up-regulated in early-stage IPF compared to normal lung tissue, and subsequently down-regulated during advanced-stage IPF. CTD-2528L19.6 was indicated to regulate fibroblast activation in IPF progression by mediating the expression of fibrosis related genes LRRC8C, DDIT4, THBS1, S100A8 and TLR7 et al. Further studies showed that silencing of CTD-2528L19.6 increases the expression of Fn1 and Collagen I both at mRNA and protein levels, promoted the transition of fibroblasts into myofibroblasts and accelerated the migration and proliferation of MRC-5 cells. In contrast, CTD-2528L19.6 overexpression alleviated fibroblast activation in MRC-5 cells induced by TGF- $\beta 1$. LnCRNA CTD-2528L19.6 inhibited fibroblast activation through regulating the expression of $\angle R R C 8 C$ in vitro assays. Our results suggest that CTD2528L19.6 may prevent the progression of IPF from early-stage and alleviate fibroblast activation during the advancedstage of IPF. Thus, exploring the regulatory effect of IncRNA CTD-2528L19.6 may provide new sights for the prevention and treatment of IPF.
\end{abstract}

\section{Introduction}

Idiopathic Pulmonary Fibrosis (IPF) is a progressive and chronic disorder that has been characterized by excessive wound repair and fibrosing interstitial pneumonia of unknown etiology ${ }^{1,2}$. IPF is likely driven by abnormal epithelium and propagated by dysregulated overabundant, heterogeneous fibroblast population in various states of

\footnotetext{
Correspondence: Yunyan Gu (guyunyan@ems.hrbmu.edu.cn) or Haihai Liang (lianghaihai@ems.hrbmu.edu.cn)

${ }^{1}$ College of Bioinformatics Science and Technology, Harbin Medical University, Harbin, China

${ }^{2}$ Department of Pharmacology (State-Province Key Laboratories of Biomedicine-Pharmaceutics of China, Key Laboratory of Cardiovascular Research, Ministry of Education), College of Pharmacy, Harbin Medical University, Harbin, China

Full list of author information is available at the end of the article

These authors contributed equally: Tingting Chen, Yingying Guo and Jiayi Wang

Edited by A. Stephanou
}

activation $^{3-5}$. It has been reported that transforming growth factor- $\beta 1$ (TGF- $\beta 1$ ) plays a key role in the development of IPF and TGF- $\beta 1$ gene polymorphisms may affect disease progression in patients with $\mathrm{IPF}^{6}$. Survival rate of the advanced-stage IPF patients at 5 years is much lower than that of early-stage $\mathrm{IPF}^{7}$. Although emerging evidence indicates that genetic studies may hold promise in the connections between early-stage and advancedstage disease ${ }^{8}$, no special study focuses on analyzing the difference of gene expression during different stages of IPF. Thus, a comprehensive understanding of the pathogenesis and progression mechanisms involved in IPF remains elusive.

Long non-coding RNAs (lncRNAs) are one type of noncoding RNAs with lengths greater than 200 nucleotides. The aberrant expression of lncRNAs has been linked to multiple biological processes involved in $\mathrm{IPF}^{9-11}$. Some

\section{(c) The Author(s) 2021}

(c) (i) Open Access This article is licensed under a Creative Commons Attribution 4.0 International License, which permits use, sharing, adaptation, distribution and reproduction cc) in any medium or format, as long as you give appropriate credit to the original author(s) and the source, provide a link to the Creative Commons license, and indicate if changes were made. The images or other third party material in this article are included in the article's Creative Commons license, unless indicated otherwise in a credit line to the material. If material is not included in the article's Creative Commons license and your intended use is not permitted by statutory regulation or exceeds the permitted use, you will need to obtain permission directly from the copyright holder. To view a copy of this license, visit http://creativecommons.org/licenses/by/4.0/. 
studies developed IPF signatures based on lncRNAs expression analysis. For example, lncRNA $H 19$ functions as a molecular sponge of miR-196a, which provides a novel therapeutic target for IPF ${ }^{12-14}$. LncRNA MALAT1, $E 2 F 1$, and $Y B X 1$ may be key regulators for the pathogenesis of IPF, in the peripheral blood of IPF patients ${ }^{15}$. LncRNA sirt1 antisense was reported to inhibit TGF- $\beta 1$ mediated epithelial-mesenchymal transition in vitro and alleviate the progression of IPF in vivo ${ }^{16}$. Crosstalk among non-coding RNAs plays a crucial regulatory role in the progression of $\mathrm{IPF}^{17}$. Savary et al. demonstrated that lncRNA DNM3OS regulates myofibroblast activation by giving rise to profibrotic mature miRNAs, such as miR$199 a-5 p / 3 p$ and $m i R-214-3 p^{18}$. Interfering with DNM3OS function not only prevents lung fibrosis but also improves established pulmonary fibrosis. LncRNA ZFAS1 promotes the progression of pulmonary fibrosis and facilitates the proliferation and phenotypic differentiation of fibroblasts into myofibroblasts via functioning as a ceRNA ${ }^{19}$, and lncRNA Hoxaas 3 promotes lung fibroblast activation and fibrosis by targeting miR-450b-5p to regulate $R u n x 1^{20}$. Huang et al. demonstrated that lncRNA FENDRR exhibits anti-fibrotic activity in pulmonary fibrosis ${ }^{21}$. Recently, we have reported several functional lncRNAs as targets for the treatment of fibrosis, and designated three pulmonary fibrosis regulatory associated lncRNAs (PFRL, PFAL, PFAR) that promote lung fibrosis by competitively binding miRNA ${ }^{22-24}$.

Previous studies provided insights into the crosstalk between mRNAs and lncRNAs to explore the regulatory mechanism in pulmonary fibrosis ${ }^{25,26}$. The changes in lncRNAs expression may affect the stability and translation of genes involved in lung fibrosis ${ }^{26}$. Hao et al. reported that lncRNA AP003419.16 regulates its adjacent gene RPS6KB2, which regulates the process of $\mathrm{IPF}^{27}$. Increasing studies suggest new theories for the pathogenesis and treatments of $\mathrm{IPF}^{25}$. Thus, systematically exploring the deregulation mechanism between lncRNAs and mRNAs in different stages of IPF will enhance our understanding of the progression of IPF.

In this study, we identified IPF pathogenic and progression related mRNAs and lncRNAs by constructing a pathogenic network and two dynamic progression networks for IPF. Our study revealed a core IPF regulatory sub-network centered on the lncRNA CTD-2528L19.6, which was up-regulated in the early-stage of IPF patients but down-regulated in advanced-stage IPF patients. In vitro assays, consistent with bioinformatics analysis of IPF progression in context: CTD-2528L19.6 was proven to alleviate fibroblast activation by regulating $L R R C 8 C$ in MRC-5 cells and the silencing CTD-2528L19.6 promoted pulmonary fibrosis. In summary, our study highlighted that CTD-2528L19.6 may prevent the progression of IPF by alleviating fibroblast activation.

\section{Results}

\section{Identification of IPF pathogenic signature and IPF progression signature}

To evaluate gene expression patterns in the occurrence and development of IPF, we identified differentially expressed (DE) genes (DEGs) for the following three comparisons: (1) early-stage IPF vs. normal; (2) advancedstage IPF $v s$. normal; (3) advanced-stage IPF $v s$. early-stage IPF. The methodological workflow for this study is summarized in Supplementary Fig. S1. Two lncRNAs and 48 mRNAs were significantly differentially expressed in all three comparisons $(P<0.01$, Student's $t$-test, Fig. 1A; Supplementary Fig. S2A). LncRNA CTD-2528L19.6, NR2F1-AS1 were significantly up-regulated in early-stage of IPF compared with normal lung and then downregulated in advanced-stage of IPF $(P<0.01$, Student's $t$ test, Fig. 1B, C). Notably, the expression level of lncRNAs CTD-2528L19.6 and NR2F1-AS1 was still higher in advanced-stage IPF than that in normal lung $(P<0.01$, Student's $t$-test, Fig. 1B, C). Also, some genes, such as DCLRE1C, S100A8, THBS1, showed reverse tendency among the normal, early-stage and advanced-stage of IPF $(P<0.01$, Student's $t$-test, Supplementary Fig. S2B-D).

Then, 43 DE lncRNAs and 835 DE mRNAs that were differentially expressed in both early-stage and advancedstage IPF compared to normal samples, were defined as IPF pathogenic signatures. IPF pathogenic lncRNAs and mRNAs showed consistently up-regulated or downregulated expression in both early-stage and advancedstage IPF compared with normal samples (Fig. 1D for lncRNAs, Supplementary Fig. S2E for mRNAs). 14 DE lncRNAs and $264 \mathrm{DE}$ mRNAs that were differentially expressed in advanced-stage IPF compared to both normal and early-stage samples, were defined as IPF progression signature. Interestingly, all IPF progression lncRNAs were sharply up-regulated in the transition from normal lung to early-stage IPF, then there was a "rebound" tendency. The expression level of IPF progression lncRNAs was obviously down-regulated during the transition to advanced-stage IPF, but was still higher compared to normal lung (Fig. 1E). A similar "rebound" tendency was observed in IPF progression mRNAs (Supplementary Fig. S2F).

Additionally, we performed pathway enrichment analyses to investigate the potential functional mechanisms involved in pathogenesis and progression of IPF. IPF pathogenic mRNAs participated in pathways related to immune system and inflammation, including "Phagosome", "Antigen processing and presentation", "Cell adhesion molecules (CAMs)", "Focal adhesion" and "ECM-receptor interaction" ( $F D R<0.05$, Hypergeometric test, Supplementary Fig. S2G). IPF progression mRNAs are involved in some IPF-related pathways, including "PI3K/Akt signaling" and immune system such as "B cell 


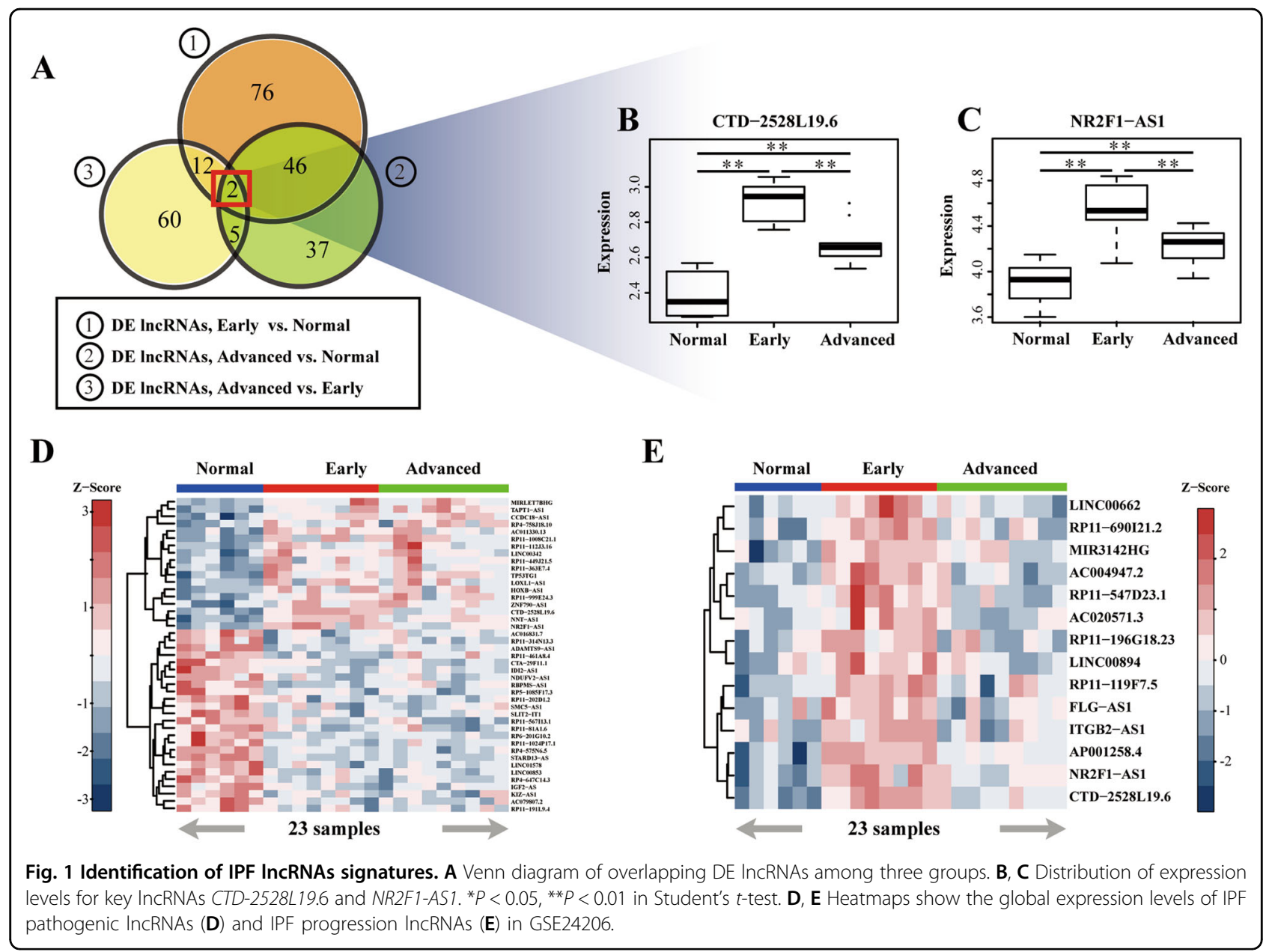

receptor signaling pathway", "Chemokine signaling pathway" and "Jak-STAT signaling pathway" $(F D R<0.05$, Hypergeometric test, Supplementary Fig. S2H). The above results indicated that dysregulation of lung fibrosis and immune systems plays important roles in $\mathrm{IPF}^{28,29}$.

\section{Analysis of IPF pathogenic co-expression network}

To capture the core lncRNA-mRNA regulatory module over pathogenesis in IPF, we constructed IPF pathogenic co-expression network (Fig. 2A). IPF pathogenic coexpression network exhibited the power-law behavior in "scale-free" network models $\left(R^{2}=0.99, P<0.01\right.$, Goodness of fit test) (Fig. 2B). The lncRNAs and mRNAs with co-expression relationships were equally distributed across the genome (Fig. 2C). A sub-network centered on CTD-2528L19.6 emerged in the IPF pathogenic coexpression network. In the dataset of GSE24206, expression of CTD-2528L19.6 was increased in early-stage of IPF and decreased in advanced-stage IPF. CTD-2528L19.6 showed a similar differential expression tendency in three independent datasets (GSE10667, SRP10849 and GSE73854, Supplementary Fig. S3A-C). Some hub
lncRNAs, such as LINC00342, RP11-1008C21.1, RP11363E7.4 and TP53TG1, may regulate fibrogenesis by regulating mRNAs CD4, CCNB1, XAF1 and PAK1 in fibroblast related gene sets, respectively (Supplementary Fig. S4). Besides, mRNAs up-regulated in the IPF pathogenic network were involved in "Primary immunodeficiency", "Axon guidance", and "T cell receptor signaling pathway" $(F D R<0.05$, Hypergeometric test, Fig. 2D). mRNAs down-regulated in the network were involved in pathways in cancer and cancer-related signaling pathways $(F D R<0.05$, Hypergeometric test, Fig. 2E).

\section{Dynamic IPF progression IncRNA-mRNA co-expression networks}

To explore the dynamic regulatory mechanism in the IPF progression, IPF early-stage specific and advancedstage specific lncRNA-mRNA co-expression networks were constructed, respectively. IPF early-stage specific network exhibited the power-law behavior in "scale-free" network models (Fig. 3A; $R^{2}=0.99, P<0.01$, Goodness of fit test, Fig. 3B), and CTD-2528L19.6 was co-expressed with 15 mRNAs in this network (Fig. 3C). IPF advanced- 


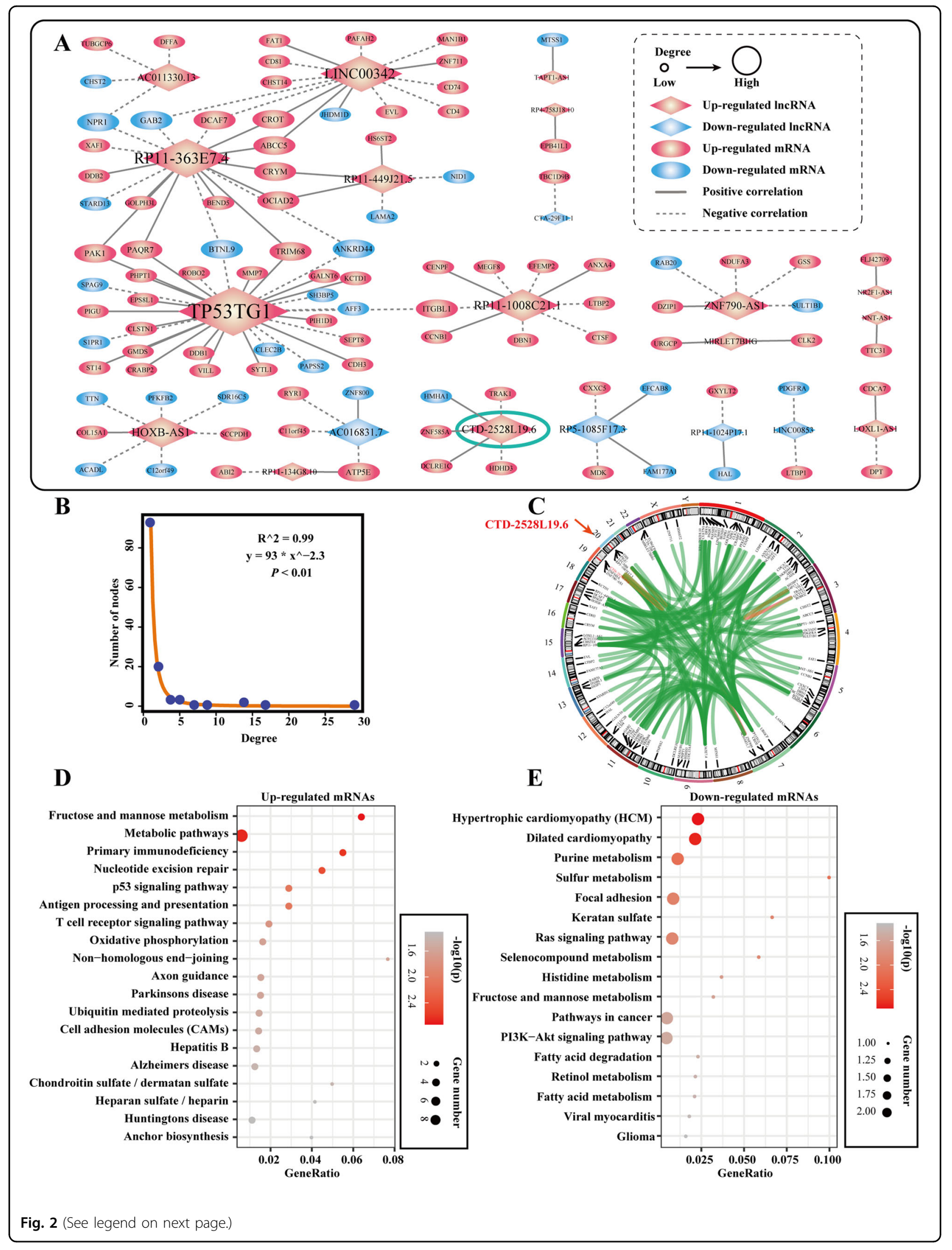


(see figure on previous page)

Fig. 2 LncRNA-mRNA co-expression networks during the pathogenesis of IPF. A IPF pathogenic IncRNAs (diamond)-mRNAs (oval) cOexpression network in IPF. The nodes marked with red color represent the up-regulated IPF pathogenic genes. The nodes marked with blue color represent the down-regulated IPF pathogenic genes. The solid lines (dotted lines) represent the positive (negative) correlation between mRNAs and IncRNAs $(|r|>0.8, P<0.01$, Pearson Correlation Test). B Distribution of the degree of genes in the IPF pathogenic network. C Circos plots display chromosomal interactions between co-expressed IncRNAs and mRNAs. The location of IncRNA CTD-2528L19.6 was marked with red arrows.

D, E KEGG pathways enrichment with IPF pathogenic up-regulated mRNAs (D) or down-regulated mRNAs (E). FDR $<0.05$, Hypergeometric test. Size of the bubble indicates the number of genes in the corresponding annotation. Color shade corresponds to the - $\log 10(p)$ value. The closer the color is to red, the more significant the enrichment is.

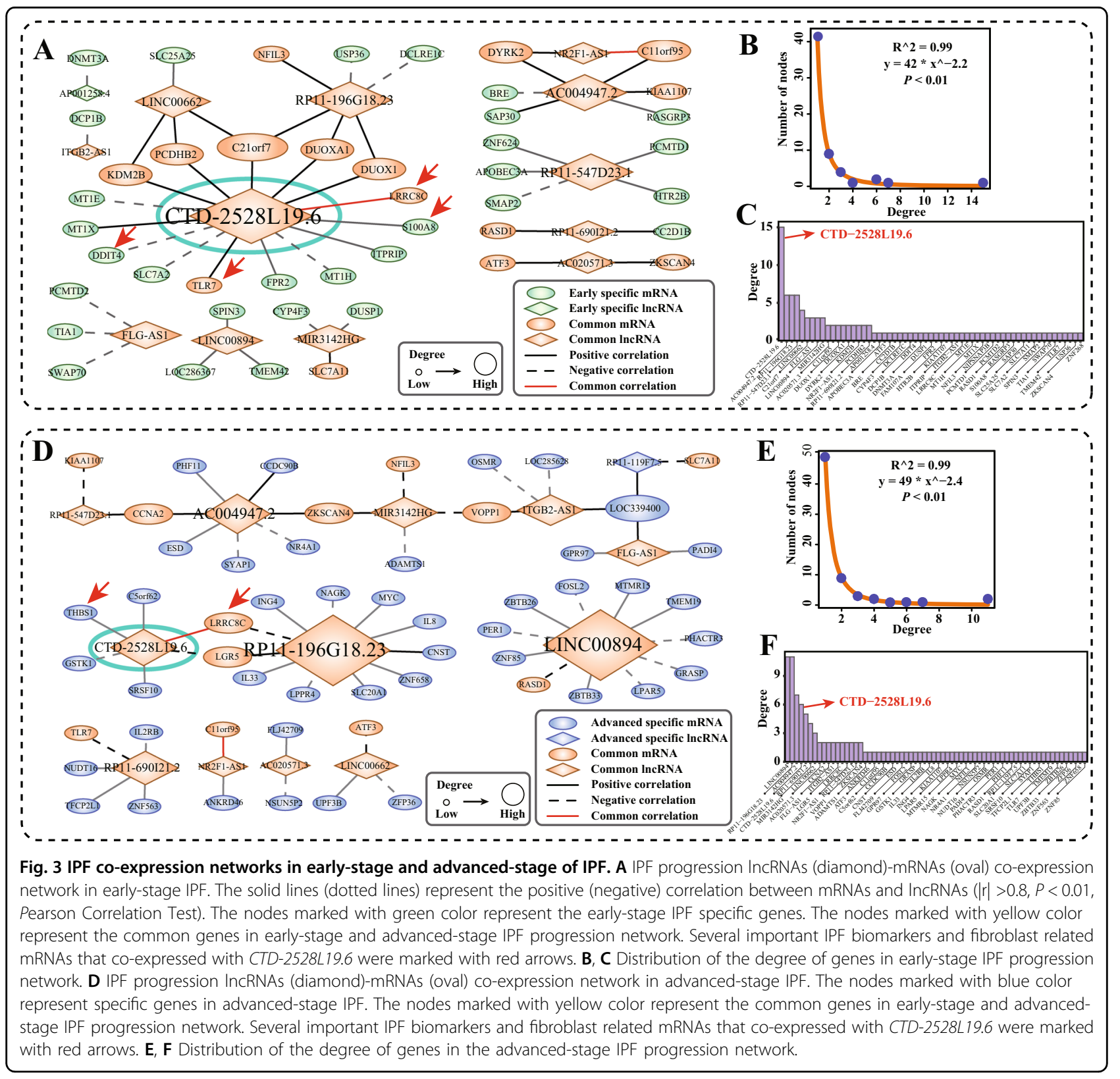

stage specific network also exhibited the power-law behavior in "scale-free" network models (Fig. 3D; $R^{2}=$ 0.99, $P<0.01$, Goodness of fit test, Fig. 3E), and CTD-
2528L19.6 was co-expressed with 6 mRNAs in this network (Fig. 3F). LRRC8C, which was correlated with CTD$2528 L 19.6$ in both networks, has been approved to be an 


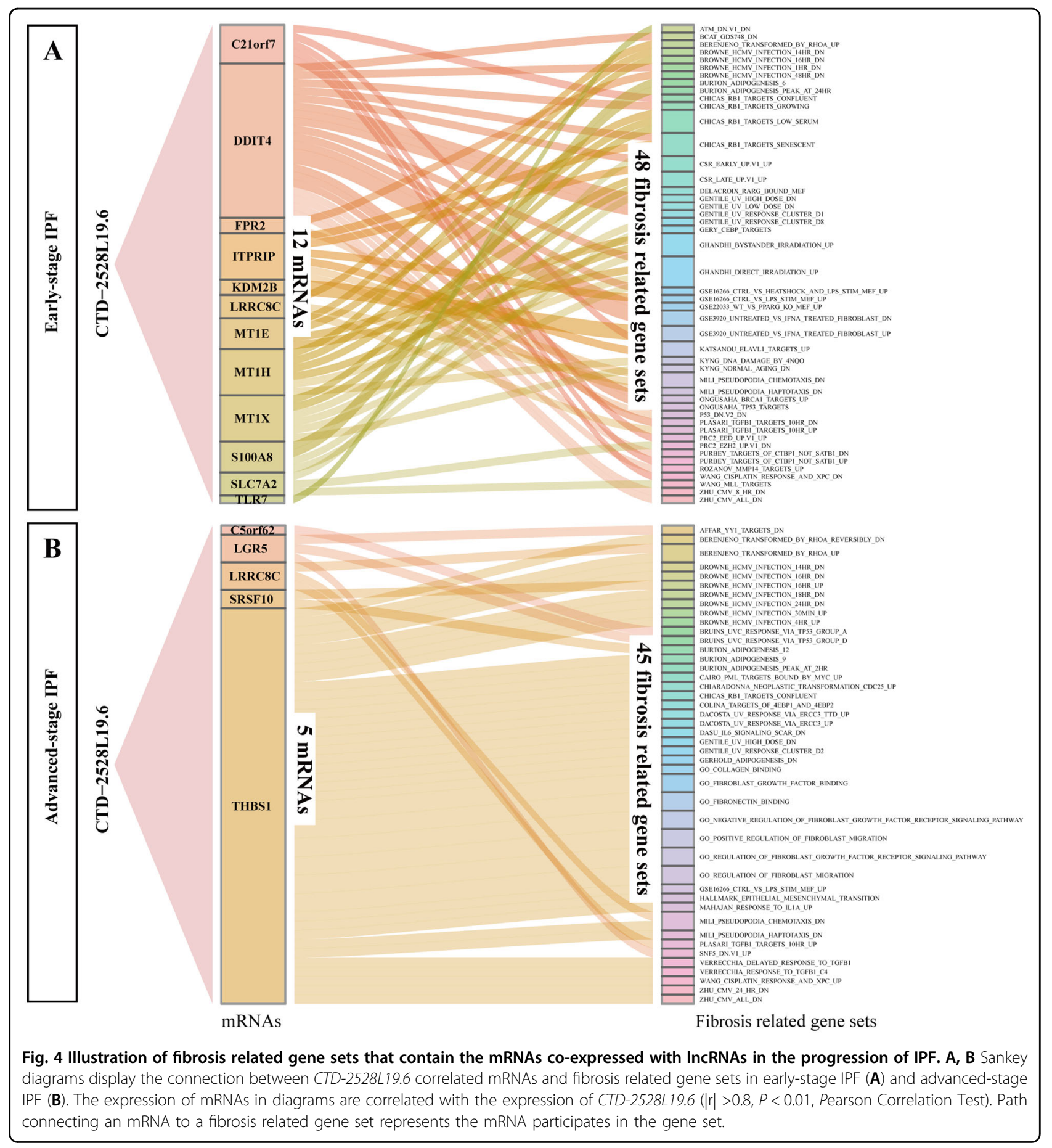

IPF biomarker ${ }^{30}$ and is involved in multiple fibrosis related gene sets (Fig. 4A, B). These results emphasized the regulatory relationship between CTD-2528L19.6 and $L R R C 8 C$ in the dynamic progression of IPF.

mRNAs whose expression correlated with CTD$2528 L 19.6$ are members of many fibrosis related biological processes. In early-stage IPF co-expression network, mRNAs S100A8 $\quad(r=0.85, \quad P=6.99 \mathrm{E}-3, \quad$ Pearson correlation test) and TLR7 ( $r=0.89, P=3.01 \mathrm{E}-3$, Pearson correlation test) that co-expressed with CTD-2528L19.6 have been reported to be novel biomarkers of IPF fibro$\operatorname{sis}^{31,32}$. DDIT4, whose expression was significantly positively co-expressed with CTD-2528L19.6 $(r=0.87, P=$ 4.24E-3, Pearson correlation test), was contained in $42 \%$ of fibrosis related gene sets, which were annotated in the MSigDB database (Fig. 4A). In advanced-stage co- 
A

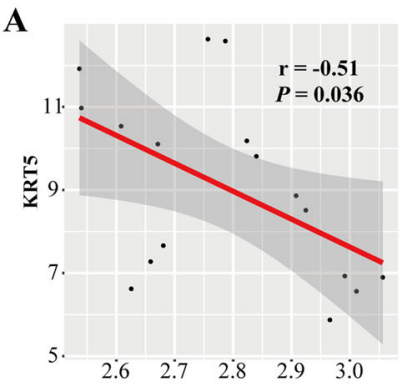

D

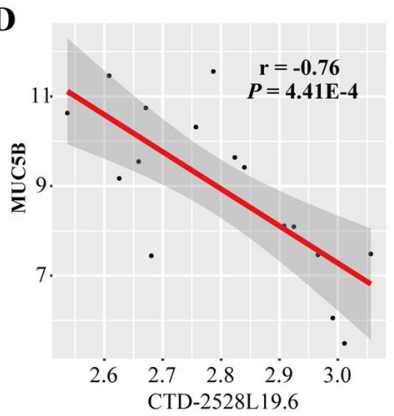

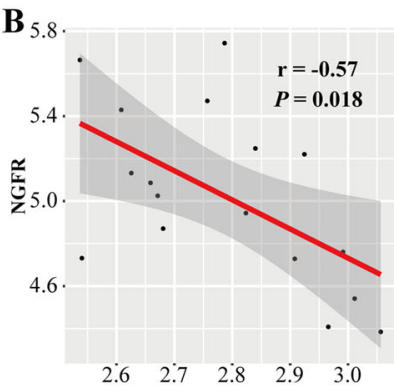

$\mathbf{E}$

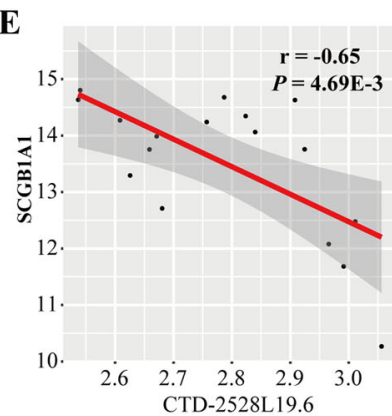

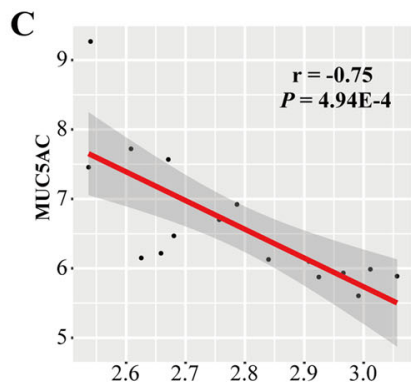

F 8.0

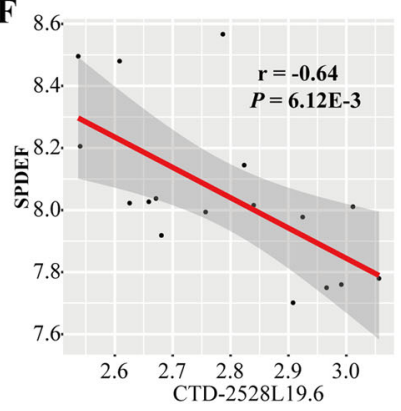

Fig. 5 Correlation between CTD-2528L19.6 and six IPF cell markers in IPF patients. A-F The correlation relationship between CTD-2528L 19.6 and six IPF cell markers (KRT5, NGFR, MUC5AC, MUC5B, SCGB1A1, SPDEF) was tested by Pearson correlation test. The fitting model was constructed using the "Im" method function in R package ggplot2.

expression network, lncRNAs RP11-196G18.23, LINC00894 and CTD-2528L19.6 were frequently coexpressed with other genes. IPF biomarker $L R R C 8 C$ was positively co-expressed with $R P 11-196 G 18.23(r=0.85, P$ $=3.67 \mathrm{E}-3$, Pearson correlation test) and CTD-2528L19.6 ( $r=0.81, P=8.71 \mathrm{E}-3$, Pearson correlation test). THBS1, which showed significant positive co-expression with CTD-2528L19.6 ( $r=0.81, P=8.7 \mathrm{E}-3$, Pearson correlation test), was contained in $84 \%$ fibrosis related gene sets (Fig. 4B). These results suggest that CTD-2528L19.6 affects lung fibrosis in early-stage and advanced-stage of IPF by regulating different fibrosis related genes.

\section{LnCRNA CTD-2528L19.6 expression is negatively correlated with fibroblast genes}

To investigate the association between CTD-2528L19.6 and fibrosis, we performed Pearson correlation coefficient test between CTD-2528L19.6 and six IPF cell markers at single-cell level ${ }^{33}$. The results from Fig. 5 showed negative correlations between CTD-2528L19.6 and IPF cell markers KRT5 $(r=-0.51, P=0.036$, Fig. 5A), NGFR $(r=$ $-0.57, P=0.018$, Fig. $5 \mathrm{~B}$ ), and strong negative correlations between CTD-2528L19.6 and IPF cell markers $M U C 5 A C(r=-0.75, P=4.94 \mathrm{E}-4$, Fig. $5 \mathrm{C}), M U C 5 B(r=$ $-0.76, P=4.41 \mathrm{E}-4$, Fig. 5D), SCGB1A1 $(r=-0.65, P=$ 4.69E-3, Fig. $5 \mathrm{E})$ and $\operatorname{SPDEF}(r=-0.64, P=6.12 \mathrm{E}-3$, Fig. $5 F)$ in IPF patients. Notably, three of the six IPF cell makers (KRT5, NGFR, MUC5B) showed significantly upregulation in advanced-stage of IPF and the other two makers (SCGB1A1, MUC5AC) were marginally significantly up-expressed in advanced-stage of IPF compared with early-stage of IPF (Supplementary Fig. S5).

\section{Silencing IncRNA CTD-2528L19.6 promotes fibroblast activation in MRC- 5 cells}

To determine the effect of IncRNA CTD-2528L19.6 in lung fibrosis, we first examined the localization of CTD2528 L19.6 in human lung fibroblast MRC-5 cells by fluorescence in situ hybridization (FISH). FISH results showed that CTD-2528L19.6 was expressed in both the nucleus and the cytoplasm (Fig. 6A). Then, we used a smart silencer against CTD-2528L19.6 (SSi-CTD) to explore the effects of CTD-2528L19.6 knockdown on the proliferation, migration and trans-differentiation of MRC5 cells (Fig. 6B). As illustrated in Fig. 6C, SSi-CTD resulted in the up-regulation of $\mathrm{Fn} 1$ and Collagen $1 \alpha 1$ at mRNA levels. In addition, silencing CTD-2528L19.6 promoted the expression of $\mathrm{Fn} 1$ and Collagen I at protein levels (Fig. 6D). Meanwhile, as illustrated in Fig. 6E-G, $\mathrm{SSi}-\mathrm{CTD}$ apparently increased the ability of cell migration, proliferation and facilitated the trans-differentiation of fibroblasts into myofibroblasts. The above results suggest that silencing lncRNA CTD-2528L19.6 can promote the activation of MRC-5 cells. 


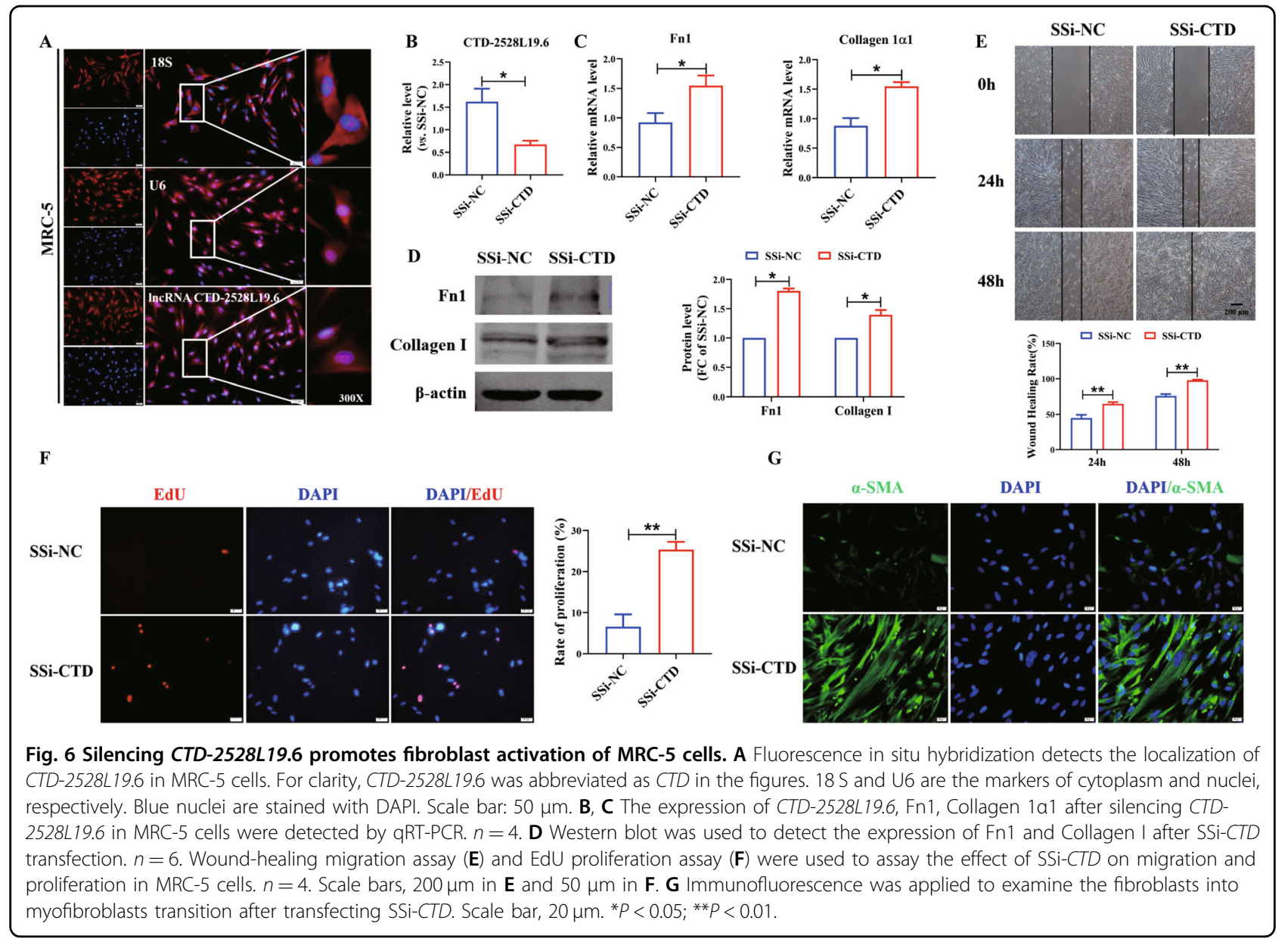

\section{Overexpression of CTD-2528L19.6 alleviates fibroblast activation induced by TGF- $\beta 1$}

Next, we transfected CTD-2528L19.6 overexpression plasmid into MRC-5 cells to determine the effect of CTD2528L19.6 on fibroblast activation (Fig. 7A). As shown in Fig. 7B, TGF- $\beta 1$ promoted the expression of Fn1 and Collagen $1 \alpha 1$, which was inhibited by overexpression of CTD-2528L19.6. Furthermore, western blot assay showed that forced expression of CTD-2528L19.6 inhibited the up-regulation of Fn 1 and Collagen I induced by TGF- $\beta 1$ at protein levels (Fig. 7C). Fibrosis occurs with abnormal activation and excessive proliferation of fibroblasts. Wound-healing migration assay showed that overexpression of CTD-2528L19.6 attenuated TGF- $\beta 1$ induced cell migration and inhibited wound healing (Fig. 7D). Through the proliferation experiment of the EdU proliferation assay, we found that overexpression of CTD2528 L19.6 suppressed the MRC-5 proliferation induced by TGF- $\beta 1$ (Fig. 7E). More importantly, we detected the expression of $\alpha$-SMA, a marker for fibroblastmyofibroblast transition, in MRC-5 cells by immunofluorescence assay. As shown in Fig. 7F, TGF- $\beta 1$ could significantly increase $\alpha$-SMA expression, whereas this effect was inhibited by the overexpression of CTD2528L19.6. These results suggest that overexpression of lncRNA CTD-2528L19.6 can suppress activation of MRC5 cells induced by TGF- $\beta 1$.

\section{Silencing $\angle R R C 8 C$ alleviated the inhibitory effect of CTD- $2528 L 19.6$ on fibroblast activation}

In the co-expression network, expression of CTD$2528 L 19.6$ was positively correlated with $L R R C 8 C$ in both early-stage $(r=0.95, P=2.82 \mathrm{E}-4$, Pearson correlation test, Fig. 3A) and advanced-stage IPF $(r=0.81, P=$ 8.71E-3, Pearson correlation test, Fig. 3D). Therefore, we performed qRT-PCR assay to examine the effect of CTD$2528 L 19.6$ on $L R R C 8 C$. Results showed that knockdown of CTD-2528L19.6 inhibited the expression of $L R R C 8 C$ in MRC-5 cells (Fig. 8A), whereas overexpression of CTD$2528 L 19.6$ promoted $L R R C 8 C$ expression at mRNA level (Fig. 8B).

Then, we constructed three small interference RNAs (siRNAs) against $L R R C 8 C$ to further explore the effects of $L R R C 8 C$ knockdown on collagen deposition. qRT-PCR 


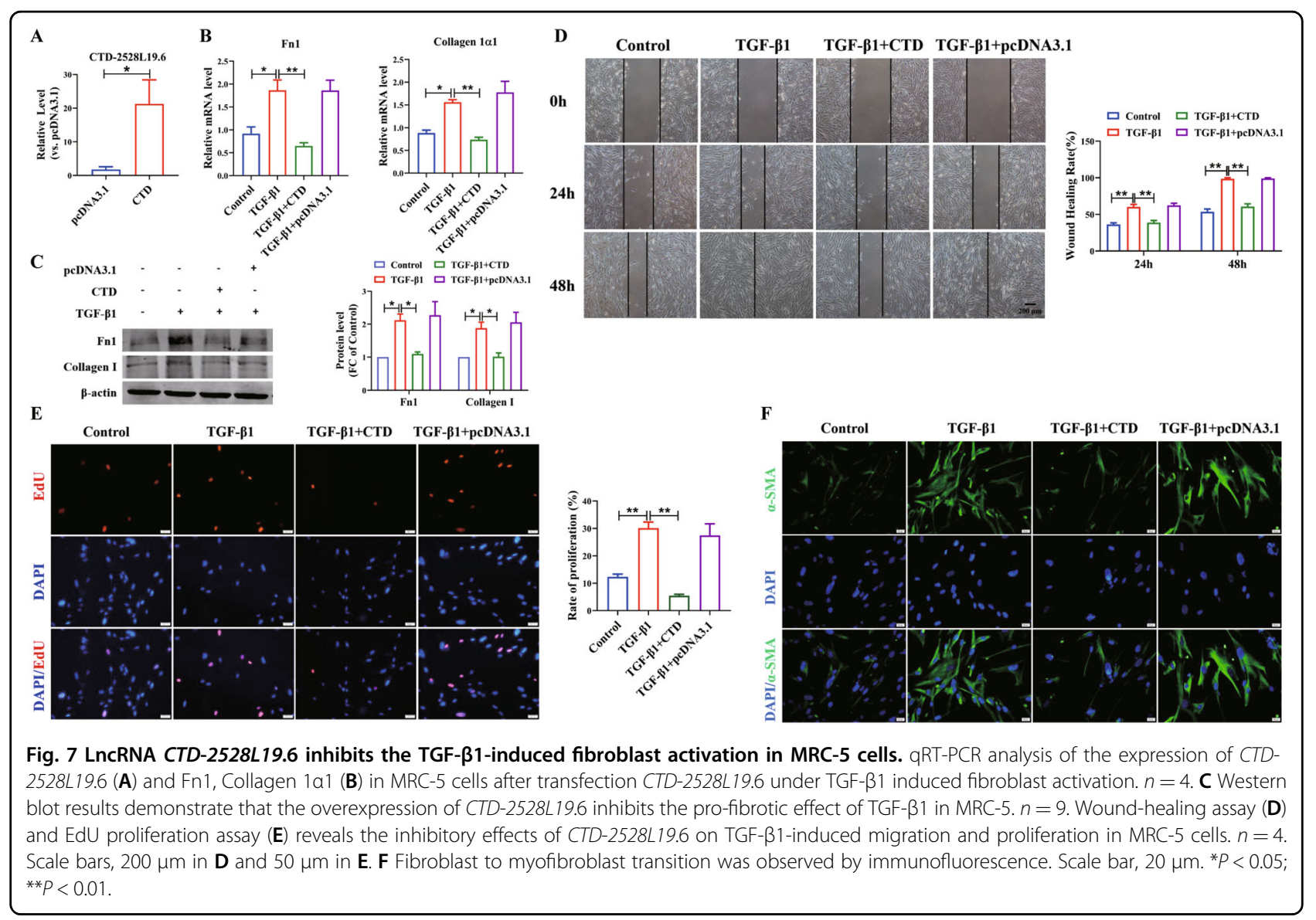

results showed that all three siRNAs could inhibit the expression of $L R R C 8 C$ in MRC-5 cells (Fig. 8C). As shown in Fig. 8D, E, silencing of $L R R C 8 C$ alleviated the inhibitory effect of CTD-2528L19.6 on Fn1 and Collagen $1 \alpha 1$ in TGF- $\beta 1$-treated MRC- 5 cells.

\section{Discussion}

In this study, by systematical analysis of transcriptome profile, we revealed a set of differentially expressed lncRNAs in IPF compared with normal lung tissues. Interestingly, some lncRNAs were up-regulated in the transition from normal lung to early-stage IPF, subsequently down-regulated during the transition to advanced-stage IPF. The IncRNA-mRNA co-expression network-based transcriptome analysis revealed a key fibrosis regulator lncRNA CTD-2528L19.6 in IPF. We demonstrated that IncRNA CTD-2528L19.6 participates in the pathogenesis and progression of IPF in different stages with different mechanisms. In MRC-5 cells, upregulation of CTD-2528L19.6 prevented the fibrosis progression of IPF by alleviating fibroblast activation. LncRNA CTD-2528L19.6 inhibited fibroblast activation through regulating the expression of $L R R C 8 C$ in vitro assays. Silencing of CTD-2528L19.6 promoted fibroblast activation, which may be a novel biomarker for IPF.

Expression of CTD-2528L19.6 was increased in earlystage of IPF and decreased in advanced-stage IPF. The dysregulation and effect of CTD-2528L19.6 in IPF is similar to the $M M P 13$, whose expression is up-regulated during two stages after fibrosis whereas knockdown of MMP13 could aggravate the progression of lung fibro$\mathrm{sis}^{34,35}$. Thus, we inferred that, at the early-stage of IPF, the adaptive up-regulating CTD-2528L19.6 is sufficiently to partially offset the activation of fibroblasts. However, with long-term stress, a sustained increase of the fibroblasts beyond a certain threshold could counteract the function of CTD-2528L19.6 up-regulation, leading to the development of fibroblast activation. Also, the biological experiments in our work revealed that enhanced expression of lncRNA CTD-2528L19.6 prevents the activation of fibroblast, which confirmed our hypothesis.

CTD-2528L19.6 may prevent the progression of IPF and exhibit anti-fibrotic activity by regulating the expression of fibrosis associated mRNAs. In IPF pathogenesis network, mRNA DCLRE1C is a member of the nonhomologous end joining pathways, and deleterious 
A

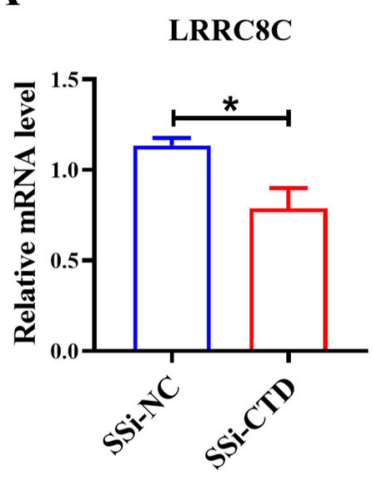

B

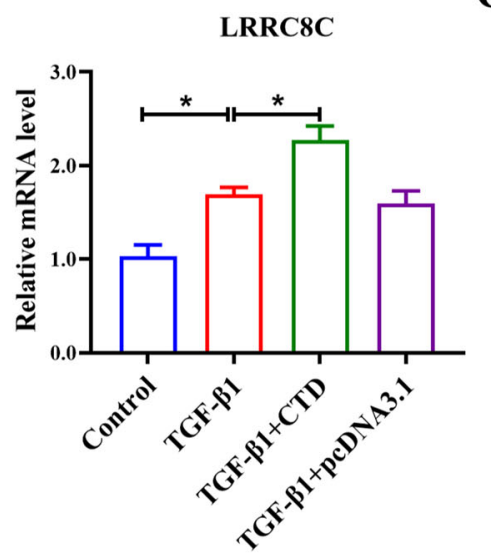

C

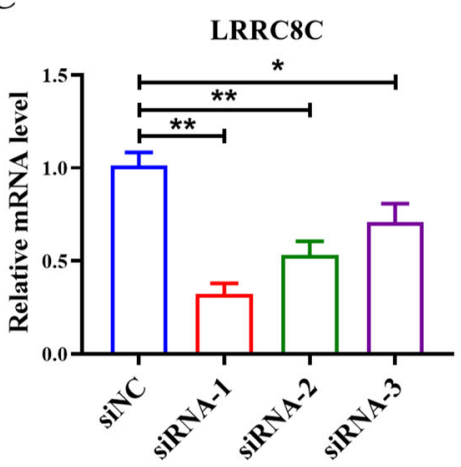

D

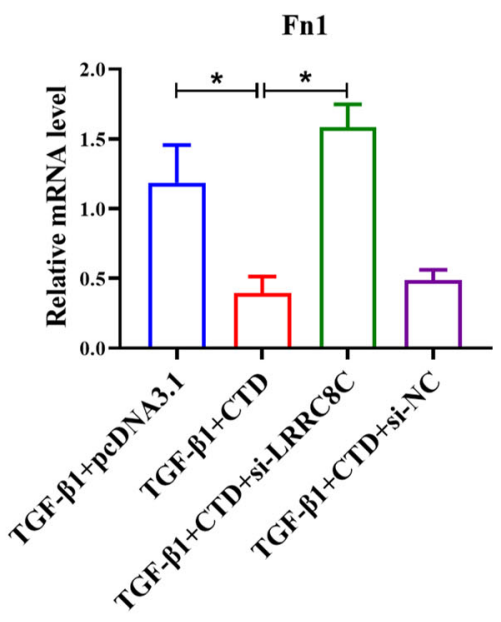

$\mathbf{E}$

Collagen $1 \propto 1$

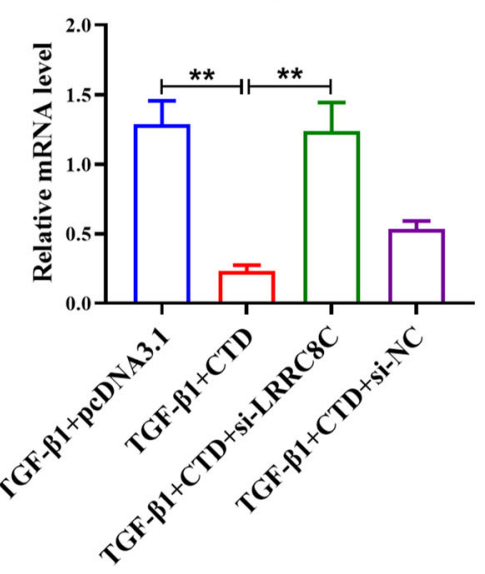

Fig. 8 LnCRNA CTD-2528L19.6 regulates LRRC8C in MRC-5 cells. A, B qRT-PCR shows that silencing or forced overexpression of CTD-2528L19.6 inhibited (A) or promoted (B) the expression of $\angle R R C 8 C$. C-E Silencing of $\angle R R C 8 C$ restored the expression levels of Fn1 and Collagen 1 a 1 in TGF- $\beta 1$ treated MRC-5 cells. $n=4 ;{ }^{*} P<0.05,{ }^{* *} P<0.01$.

mutations which can cause severe combined immunodeficiency ${ }^{36}$. In early-stage IPF progression networks, mRNA S100A8 has been reported in bronchoalveolar lavage fluid as a biomarker of IPF fibrosis ${ }^{31}$. In advancedstage IPF progression network, mRNA THBS1 is coexpressed with CTD-2528L19.6, which activates the reproduction of fibroblast cells in mice ${ }^{37}$. TLR7 has been revealed to be a novel IPF associated gene and biomarker ${ }^{32}$, and is dysregulated in IPF pathogenesis ${ }^{38}$. In IPF progression network, genes S100A8 and TLR7, which were positively correlated with the expression of CTD2528L19.6, act as immune cell markers ${ }^{32}$. The crosstalk analysis between mRNAs and lncRNAs highlights the important role of immune regulation in IPF, which warrants further detail in vitro or in vivo biological experiments. The functional relationship between $L R R C 8 C$ and CTD-2528L19.6 in fibrosis was proved in vitro assays. LncRNA CTD-2528L19.6 inhibited fibroblast activation through regulating the expression of $L R R C 8 C$. Limited by the sample amount of IPF data set, we did not control the FDR when performing differential expression analysis. However, the roles of CTD-2528L19.6 in IPF predicted by bioinformatics analysis could be validated by cellular experiments and some correlated genes were proved to be involved in IPF by functional annotation. Another key IncRNA NR2F1-AS1 with many transcripts was not tested in this study, which is worthy of our follow-up in-depth research. Notably, the lncRNA TP53TG1, which is most frequently co-expressed with mRNAs in IPF pathogenesis network, also deserves our following study.

Overall, we predicted IPF pathogenic and progression related lncRNAs and mRNAs through analyzing transcriptional profile, then constructed an IPF pathogenic IncRNA-mRNA co-expression network and dynamic IPF progression networks. A key lncRNA CTD-2528L19.6 was captured to regulate the pathogenesis and progression of IPF as a potential biomarker. CTD-2528L19.6 may regulate fibroblast activation in IPF progression by mediating 
the expression of some mRNAs, such as LRRC8C, DDIT4, THBS1, S100A8 and TLR7. Our results suggest that during early-stage, CTD-2528L19.6 was up-regulated to prevent IPF, while CTD-2528L19.6 was down-regulated to promote fibroblast activation from early-stage to advanced-stage transition. This finding provides a framework for designing interventions that could prevent the development or progression of fibrosis at various stages of IPF development. The present research also reveals some lncRNAs as potential IPF biomarkers.

\section{Materials and methods}

\section{Microarray data and processing}

Gene expression dataset (GSE24206) ${ }^{39}$, with 17 IPF (8 early-stage IPF and 9 advanced-stage IPF) and 6 normal lung tissues, was downloaded from the Gene Expression Omnibus (GEO, http://www.ncbi.nlm.nih.gov/geo/) (Supplementary Fig. S1A). Expression for the Affymetrix U133 Plus 2.0 GeneChips was estimated by robust multiarray average then $\log 2$ transformed. Data were filtered prior to analysis: If a gene was mapped to multiple probe sets, the expression value for the gene was generated by averaging. Probes that did not map to any Gene ID or map to multiple Gene IDs were deleted ${ }^{40}$.

GSE10667 and GSE73854 were collected from the GEO and SRP10849 was download from The Sequence Read Archive (SRA, https://www.ncbi.nlm.nih.gov/sra/).

\section{Identification of IPF signature}

We performed three comparisons using Student's $t$-test: (1) early-stage IPF vs. normal; (2) advanced-stage IPF vs. normal; (3) advanced-stage IPF $v s$. early-stage IPF. We selected genes with $P<0.01$ as DEGs, including DE lncRNAs and DE mRNAs. The DEGs derived from both (1) and (2) were defined as IPF pathogenic signatures. And, the DEGs detected from both (1) and (3) were defined as IPF progression signatures, as showed in Supplementary Fig. S1B.

\section{Co-expression network construction}

Pearson correlation test was used to test the expression correlation between differential expressed lncRNAs and mRNAs. The lncRNA-mRNA pairs with the absolute value of correlation coefficient $r>0.8$ and $P$-value $<0.01$ were included in the co-expression network.

Then, a pathogenic co-expression network between IPF pathogenic lncRNAs and mRNAs was constructed in IPF samples. Two IPF progression co-expression networks between IPF progression lncRNAs and mRNAs were constructed in early-stage IPF and advanced-stage IPF samples, respectively (Supplementary Fig. S1C).

\section{Fibrosis related gene sets and IPF cell markers}

Fibrosis related gene sets were collected from the Molecular Signatures Database (MSigDB, https://www. gsea-msigdb.org/gsea/msigdb), including fibroblast related gene sets. These annotated sets of genes involved in fibrosis related biochemical pathways, signaling cascades, expression profiles from research publications, and other biological concepts ${ }^{41}$. IPF cell markers (KRT5, MUC5AC, $M U C 5 B$, NGFR, SCGB1A1, SPDEF) were collected from the CellMarker database (http://biocc.hrbmu.edu.cn/ CellMarker $/)^{42}$.

\section{Pathway enrichment analysis}

Pathway information was downloaded from Kyoto Encyclopedia of Genes and Genomes (KEGG) on August 17, 2017 (http://www.genome.jp/kegg/), consists of six top categories (09100 to 09160) for KEGG pathway maps. The hypergeometric distribution model was used to test whether KEGG pathways were enriched with genes in the co-expression network. The $P$-value was adjusted by the Benjamini-Hochberg $(\mathrm{BH})$ procedure. The pathways with a false discovery rate (FDR) less than 0.05 were considered to be significant.

\section{MRC- 5 cell culture and transfection}

MRC-5 cell line was purchased from Cell Bank of Chinese Academy of Sciences, and cultured in 89\% DMEM (Biological Industries, Israel) containing 10\% FBS (Biological Industries, Cromwell, CT, USA) and 1\% PenicillinStreptomycin-Amphotericin B (Solarbio, China) then plated in a standard humidity incubator at $37^{\circ} \mathrm{C}$ with $5 \%$ $\mathrm{CO}_{2}$. PCR was used to synthesize the full-length of lncRNA CTD-2528L19.6 and inserted into the pcDNA3.1 vector. pcDNA3.1 empty vector was used as control. LncRNA smart silencer SSi-CTD/SSi-NC (negative control) was constructed by RiboBio Tech (Guangzhou, China). CTD-2528L19.6 plasmid or SSi-CTD/SSi-NC and Lipofectamine 2000 (Invitrogen, Carlsbad, CA, U.S.A.) transfection reagents were mixed with Opti-MEM (GIBCO, Grand Island, NY, U.S.A.) in serum-free medium, respectively. Then incubated for $5 \mathrm{~min}$ and protected from the light. Mixed two liquids and placed for $15 \mathrm{~min}$ at room temperature, then added to the cell plate and cultured in incubator. After transfection $6 \mathrm{~h}$ replaced with a normal culture medium. After cells were cultured with $10 \mathrm{ng} / \mathrm{ml}$ TGF- $\beta 1$ (Sigma-Aldrich, U.S.A.) for $48 \mathrm{~h}$ and prepared for further analysis (Supplementary Fig. S1D).

\section{Fluorescence in situ hybridization}

Fluorescence in situ hybridization (FISH) was performed using the lncRNA FISH Probe Mix kit (Ribobio, Guangzhou, China). MRC-5 cells were fixed in 4\% paraformaldehyde (Solarbio, China) for $10 \mathrm{~min}$ and then added $1 \mathrm{ml}$ precooling permeable solution $(5 \mu \mathrm{l}$ Triton-X $+1 \mathrm{ml} \mathrm{PBS})$ for $5 \mathrm{~min}$ at $4{ }^{\circ} \mathrm{C}$. Added $200 \mu \mathrm{l}$ prehybridization buffer into each well then incubated at $37^{\circ} \mathrm{C}$ for $30 \mathrm{~min}$. Then discarded the pre-hybridization 
buffer and added $150 \mu$ hybridization buffer with lncRNA FISH Probe Mix, hybridized overnight at $37^{\circ} \mathrm{C}$. Later washed with buffer I (4x SSC), buffer II ( $2 x$ SSC) and buffer III (1x SSC) at $42^{\circ} \mathrm{C}$. The nuclei were stained with DAPI (Roche Molecular Biochemicals, Basel, Switzerland). The images were taken under the inverted fluorescence microscope (Olympus, IX73, Japan).

\section{RNA extraction and quantitative RT-PCR}

The total RNA of cells was extracted with TRIzol reagent. The concentration and purity of extracted RNA were determined by NanoDrop 8000 (Thermo, U.S.A). RNA reverse transcription for cDNA by using $5 \times$ All-inOne RT Master Mix. cDNA was used to detect the relative expression of mRNA by real-time quantitative reverse transcriptase polymerase chain reaction (qRT-PCR) in the presence of SYBR Green fluorescent dye (Applied Biosystems, Foster City, CA). The relative expression level was calculated based on the Ct values and GAPDH was used as a normalized control.

\section{Protein extraction and Western blot}

Total proteins of MRC-5 cells were extracted and lysed with RIPA buffer (Beyotime, Jiangsu, China) containing protease inhibitor. Protein samples were separated on $8 \%$ sodium dodecyl sulfate-polyacrylamide gel and transferred to pure nitrocellulose (Pall Life Sciences, Ann Arbor, MI, USA) after electrophoresis. The membranes were probed with primary antibodies against $\beta$-actin (1:500, 66009-1-Ig, Proteintech, Wuhan, China), Fn1 (1:500, 15613-1-AP, Proteintech, Wuhan, China) and Collagen I (1:500, WL0088, Wanlei, Liaoning, China). The protein expression levels were detected by the Odyssey Infrared Imaging System (Odyssey CLX, Biosciences, USA).

\section{Immunofluorescence staining}

After transfected and treated with TGF- $\beta 1$, MRC- 5 cells were fixed by $4 \%$ paraformaldehyde at room temperature for $30 \mathrm{~min}$, and then permeabilized with $0.1 \%$ Triton X100. Then, the cells were blocked with $50 \%$ goat serum and incubated with anti-Rabbit $\alpha$-SMA antibody (1:100, ab7817, Abcam). Finally, cells were incubated with antirabbit IgG $(\mathrm{H}+\mathrm{L})$ (Alexa Fluor 488 Conjugate) (1:500, 4412, CST). Nuclei were stained with DAPI. Immunofluorescence images were photographed under fluorescence microscope.

\section{EdU cell proliferation assay}

According to the Cell-Light EdU DNA cell proliferation kit (RiboBio, Guangzhou, China) instructions, the cells were incubated with $200 \mu \mathrm{l}, 50 \mu \mathrm{M}$ EdU solution. The cells were fixed with $4 \%$ paraformaldehyde and incubated in a $2 \mathrm{mg} / \mathrm{ml}$ glycine shaker. Afterwards, cells were stained with Apollo solution staining for proliferating cells. Nuclei were stained by DAPI. Images were finally photographed under the inverted fluorescence microscope.

\section{Wound-healing migration assay}

Wound-healing migration assay was performed as previously described ${ }^{24}$. MRC-5 cells were seeded in 6-well plates and grown until formation of confluent monolayer. Then cells were gently scratched with a $10 \mu$ pipette tip. The scratch healing areas were observed and photographed under microscope ( $\times 20$ objective). Afterwards, the cells were transfected and added with TGF- $\beta 1$. The images were taken by the Nikon Ts100 microscope (Nikon, Tokyo, Japan) and analyzed by using Image-J. First, the images resolution was changed to 8-bit and adjust the contrast of the enhanced image. After that, smoothed the scratch edge then found edge again. Finally, set the appropriate image threshold and measured the scratch area. Wound healing rate $(\%)=1-(24 \mathrm{~h}$ or $48 \mathrm{~h}$ scratch area $/ 0 \mathrm{~h}$ scratch area)* 100 .

\section{Statistics and analysis}

Student's $t$-test was used for statistical analysis between two groups. The fitting regression was constructed to modeling the relationship between CTD-2528L19.6 and IPF cell markers with linear fitting using formula $=\mathrm{y} \sim \mathrm{x}$ with method = "lm" in R package ggplot2. All bioinformatics analyses were carried out using $\mathrm{R}$ software version 3.6.0 (http://www.r-project.org/). Cytoscape software (version 3.6.0) was used to visualize the IncRNA-mRNA co-expression networks. All experimental data were presented as mean \pm SEM. One-way analysis of variance (ANOVA) was used to determine the statistically significant differences among multiple groups. Statistical analyses were carried out using GraphPad Prism 8.0.

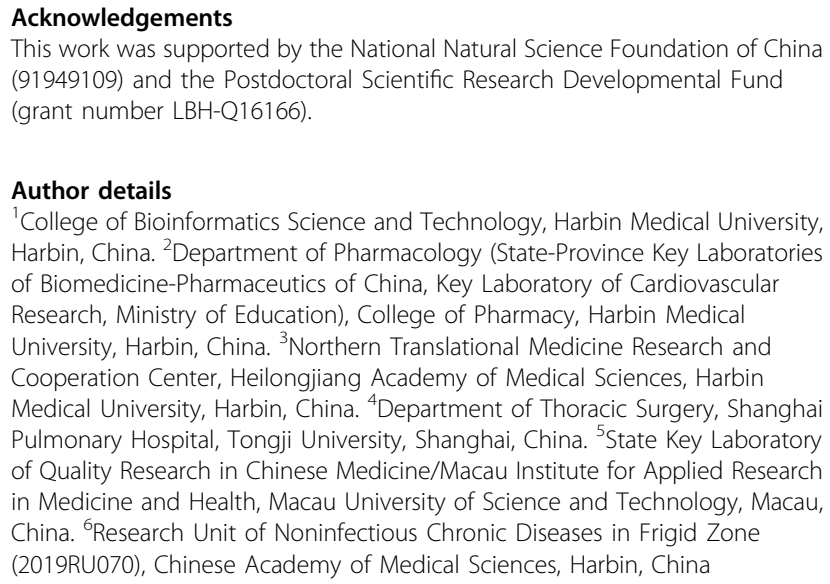

\section{Author details}

${ }^{1}$ College of Bioinformatics Science and Technology, Harbin Medical University, Harbin, China. ${ }^{2}$ Department of Pharmacology (State-Province Key Laboratories of Biomedicine-Pharmaceutics of China, Key Laboratory of Cardiovascular Research, Ministry of Education), College of Pharmacy, Harbin Medical University, Harbin, China. ${ }^{3}$ Northern Translational Medicine Research and Cooperation Center, Heilongjiang Academy of Medical Sciences, Harbin Medical University, Harbin, China. ${ }^{4}$ Department of Thoracic Surgery, Shanghai Pulmonary Hospital, Tongji University, Shanghai, China. ${ }^{5}$ State Key Laboratory of Quality Research in Chinese Medicine/Macau Institute for Applied Research in Medicine and Health, Macau University of Science and Technology, Macau, China. ${ }^{6}$ Research Unit of Noninfectious Chronic Diseases in Frigid Zone

(2019RU070), Chinese Academy of Medical Sciences, Harbin, China

\section{Author contributions}

Y.G. and H.L. designed the research; T.C. and Y.G. wrote the draft; Y.G. and H.L. revised the manuscript; T.C. and L.A. performed the bioinformatics analysis. Y.G., J.W., L.M., W.H., Z.L., X.Y., J.L. and X.F. performed cellular experiments. All authors read and approved the final manuscript. 


\section{Data availability}

The datasets analyzed during the current study are available from the public databases.

\section{Conflict of interest}

The authors declare no competing interests.

\section{Publisher's note}

Springer Nature remains neutral with regard to jurisdictional claims in published maps and institutional affiliations.

Supplementary information The online version contains supplementary material available at https://doi.org/10.1038/s41419-021-03884-5.

Received: 24 February 2021 Revised: 25 May 2021 Accepted: 27 May 2021 Published online: 10 June 2021

\section{References}

1. King, T. E. Jr et al. All-cause mortality rate in patients with idiopathic pulmonary fibrosis. Implications for the design and execution of clinical trials. Am. J. Respir. Crit. Care Med. 189, 825-31 (2014).

2. Kinoshita, T. \& Goto, T. Molecular mechanisms of pulmonary fibrogenesis and its progression to lung cancer: a review. Int. J. Mol. Sci. 20, 1461 (2019).

3. King, T. E. Jr, Pardo, A. \& Selman, M. Idiopathic pulmonary fibrosis. Lancet $\mathbf{3 7 8}$ 1949-61 (2011).

4. Lederer, D. J. \& Martinez, F. J. Idiopathic pulmonary fibrosis. N. Engl. J. Med. 378, 1811-23 (2018).

5. Selman, M. \& Pardo, A. The leading role of epithelial cells in the pathogenesis of idiopathic pulmonary fibrosis. Cell Signal 66, 109482 (2020).

6. Jiang, D. \& Liang, J. A long noncoding RNA links TGF-beta signaling in lung fibrosis. Am. J. Respir. Crit. Care Med. 200, 123-5 (2019).

7. Scelfo, C., Caminati, A. \& Harari, S. Recent advances in managing idiopathic pulmonary fibrosis. F1000Res. 6, 2052 (2017).

8. Putman, R. K., Rosas, I. O. \& Hunninghake, G. M. Genetics and early detection in idiopathic pulmonary fibrosis. Am. J. Respir. Crit. Care Med. 189, 770-8 (2014).

9. Di Mauro, S., et al. Circulating coding and long non-coding RNAs as potential biomarkers of idiopathic pulmonary fibrosis. Int. J. Mol. Sci. 21, 8812 (2020).

10. Hadjicharalambous, M. R. \& Lindsay, M. A. Idiopathic pulmonary fibrosis: pathogenesis and the emerging role of long non-coding RNAs. Int. J. Mol. Sci. 21, 524 (2020).

11. Liu, P. et al. The NRF2-LOC344887 signaling axis suppresses pulmonary fibrosis. Redox Biol. 38, 101766 (2020)

12. Lu, Q. et al. The InCRNA H19 mediates pulmonary fibrosis by regulating the miR-196a/COL1A1 axis. Inflammation 41, 896-903 (2018).

13. Omura, J. et al. Identification of long noncoding RNA H19 as a new biomarker and therapeutic target in right ventricular failure in pulmonary arterial hypertension. Circulation 142, 1464-84 (2020).

14. Xiao, T. et al. LncRNA H19-mediated M2 polarization of macrophages promotes myofibroblast differentiation in pulmonary fibrosis induced by arsenic exposure. Environ. Pollut. 268, 115810 (2021).

15. Wang, F., Li, P. \& Li, F. S. Integrated analysis of a gene correlation network identifies critical regulation of fibrosis by IncRNAs and TFs in idiopathic pulmonary fibrosis. Biomed. Res. Int. 2020, 6537462 (2020).

16. Qian, W., Cai, X. \& Qian, Q. Sirt1 antisense long non-coding RNA attenuates pulmonary fibrosis through sirt1-mediated epithelial-mesenchymal transition. Aging 12, 4322-36 (2020).

17. Wang, Y. et al. Decrypting the crosstalk of noncoding RNAs in the progression of IPF. Mol. Biol. Rep. 47, 3169-79 (2020).

18. Savary, G. et al. The long noncoding RNA DNM3OS is a reservoir of FibromiRs with major functions in lung fibroblast response to TGF-beta and pulmonary fibrosis. Am. J. Respir. Crit. Care Med. 200, 184-98 (2019).
19. Yang, Y. et al. IncRNA ZFAS1 promotes lung fibroblast-to-myofibroblast transition and ferroptosis via functioning as a ceRNA through miR-150-5p/ SLC38A1 axis. Aging 12, 9085-102 (2020).

20. Lin, S. et al. LncRNA Hoxaas3 promotes lung fibroblast activation and fibrosis by targeting miR-450b-5p to regulate Runx1. Cell Death Dis. 11, 706 (2020).

21. Huang, C. et al. Long noncoding RNA FENDRR exhibits antifibrotic activity in pulmonary fibrosis. Am. J. Respir. Cell Mol. Biol. 62, 440-53 (2020).

22. Jiang, $\mathrm{H}$. et al. Inhibition of IncRNA PFRL prevents pulmonary fibrosis by disrupting the miR-26a/smad2 loop. Am. J. Physiol. Lung Cell Mol. Physiol. 315, L563-L575 (2018).

23. Li, X. et al. IncRNA PFAL promotes lung fibrosis through CTGF by competitively binding miR-18a. FASEB J. 32, 5285-97 (2018).

24. Zhao, X. et al. IncRNA PFAR promotes lung fibroblast activation and fibrosis by targeting miR-138 to regulate the YAP1-twist axis. Mol. Ther. 26, 2206-17 (2018).

25. Li, C. et al. Crosstalk of mRNA, miRNA, IncRNA, and circRNA and their regulatory pattern in pulmonary fibrosis. Mol. Ther. Nucleic Acids 18, 204-18 (2019).

26. Zhou, Y. et al. Integrated analysis of IncRNA and mRNA transcriptomes reveals new regulators of ubiquitination and the immune response in silica-induced pulmonary fibrosis. Biomed. Res. Int. 2019, 6305065 (2019).

27. Hao, X., Du, Y., Qian, L., Li, D. \& Liu, X. Upregulation of long noncoding RNA AP003419.16 predicts high risk of agingassociated idiopathic pulmonary fibrosis. Mol. Med. Rep. 16, 8085-91 (2017).

28. Kolahian, S., Fernandez, I. E., Eickelberg, O. \& Hartl, D. Immune mechanisms in pulmonary fibrosis. Am. J. Respir. Cell Mol. Biol. 55, 309-22 (2016).

29. Zhang, X. L., Xing, R. G., Chen, L., Liu, C. R. \& Miao, Z. G. PI3K/Akt signaling is involved in the pathogenesis of bleomycininduced pulmonary fibrosis via regulation of epithelialmesenchymal transition. Mol. Med. Rep. 14, 5699-706 (2016).

30. Huang, Yong (Dyer, IN, US), Herazo-maya, Jose David (North Haven, CT, US), Kaminski, Naftali (New Haven, CT, US), Gibson, Kevin (Gibsonia, PA, US), Garcia, Joe G. N. (Tucson, AZ, US), inventor The University of Chicago (Chicago, IL, US), The Board of Trustees of the University of Illinois (Urbana, IL, US),University of Pittsburgh - Of the Commonwealth System of Higher Education (Pittsburgh, PA, US), assignee. BIOMARKERS FOR ASSESSING IDIOPATHIC PULMONARY FIBROSIS. U.S. patent No. 10,036,069. 2018 Jul. 31.

31. Hara, A. et al. S100A9 in BALF is a candidate biomarker of idiopathic pulmonary fibrosis. Respir. Med. 106, 571-80 (2012).

32. Wang, Y. et al. Unsupervised gene expression analyses identify IPF-severity correlated signatures, associated genes and biomarkers. BMC Pulm. Med. 17, 133 (2017).

33. $\mathrm{Xu}$, Y. et al. Single-cell RNA sequencing identifies diverse roles of epithelial cells in idiopathic pulmonary fibrosis. JCI Insight 1, e90558 (2016).

34. Cabrera, S. et al. Delayed resolution of bleomycin-induced pulmonary fibrosis in absence of MMP13 (collagenase 3). Am. J. Physiol. Lung Cell Mol. Physiol. 316, L961-L976 (2019).

35. Nkyimbeng, T. et al. Pivotal role of matrix metalloproteinase 13 in extracellular matrix turnover in idiopathic pulmonary fibrosis. PLoS ONE 8, e73279 (2013).

36. Sundin, M., Marits, P., Ramme, K., Kolios, A. G. A. \& Nilsson, J. Severe combined immunodeficiency (SCID) presenting in childhood, with agammaglobulinemia, associated with novel compound heterozygous mutations in DCLRE1C. Clin. Immunol. 200, 16-18 (2019).

37. Mouton, A. J. et al. Fibroblast polarization over the myocardial infarction time continuum shifts roles from inflammation to angiogenesis. Basic Res. Cardiol. 114, 6 (2019).

38. Krafft, E. et al. Transforming growth factor beta 1 activation, storage, and signaling pathways in idiopathic pulmonary fibrosis in dogs. J. Vet. Intern. Med. 28, 1666-75 (2014).

39. Meltzer, E. B. et al. Bayesian probit regression model for the diagnosis of pulmonary fibrosis: proof-of-principle. BMC Med. Genomics 4, 70 (2011).

40. Dai, M. et al. Evolving gene/transcript definitions significantly alter the interpretation of GeneChip data. Nucleic Acids Res. 33, e175 (2005).

41. Liberzon, A. et al. Molecular signatures database (MSigDB) 3.0. Bioinformatics 27, 1739-40 (2011)

42. Zhang, $X$. et al. CellMarker: a manually curated resource of cell markers in human and mouse. Nucleic Acids Res. 47, D721-D728 (2019). 